MRS Advances (C) 2017 Materials Research Society

DOI: 10.1557/adv.2017.267

\title{
Advanced anode materials for sodium ion batteries: carbodiimides
}

\author{
Aitor Eguia-Barrio ${ }^{1}$, Elizabeth Castillo-Martinez ${ }^{2, \#, *}$, Xiaohui Liu $^{3}$, Richard Dronskowski ${ }^{3}$, Luis \\ Lezama $^{1}$, Michel Armand ${ }^{2}$ and Teofilo Rojo ${ }^{1,2, *}$ \\ ${ }^{1}$ Dpto. de Química Inorgánica, Universidad del País Vasco UPV/EHU, P.O. Box 644, 48080 \\ Bilbao, Spain. \\ ${ }^{2}$ CIC Energigune, Parque Tecnológico de Alava, C/Albert Einstein 48, 01510, Miñano, Spain \\ ${ }^{\#}$ Current address: Department of Chemistry, University of Cambridge, Lensfield Road, CB2 \\ 1EW, Cambridge, UK. \\ ${ }^{3}$ Institute of Inorganic Chemistry, RWTH Aachen University, Landoltweg 1, D-52056, Aachen, \\ Germany \\ *Corresponding author: ilaisza@hotmail.com,trojo@cicenergigune.com
}

\begin{abstract}
TMNCN (where $\mathrm{TM}=\mathrm{Mn}^{2+}, \mathrm{Fe}^{2+}, \mathrm{Co}^{2+}$ or $\mathrm{Ni}^{2+}$ ) have been recently proposed as electrochemically active materials for $\mathrm{Na}$-ion insertion that operate via conversion reaction. Their electrochemical performance for Na-ion batteries is presented here with an emphasis on long-term cycling. With a very low voltage for $\mathrm{Na}$ insertion of $\sim 0.1 \mathrm{~V} v s \mathrm{Na}^{+} / \mathrm{Na}$ for $\mathrm{MnNCN}$, the overpotential observed in batteries of $\mathrm{MnNCN}$ plays a very important role in their performance, evidencing big differences in the electrochemical performance between materials produced with different nano- and micrometer particle sizes evidenced by SEM images. A more suitable voltage for the conversion reaction accompanied by less overpotential is shown by FeNCN, CoNCN and NiNCN. Despite the lower reversible capacity achieved by FeNCN (450 $\mathrm{mAh} / \mathrm{g}$ ) in comparison with $\mathrm{CoNCN}$ and $\mathrm{NiNCN}$ in the first cycle; the smallest first-cycle irreversible capacity $(220 \mathrm{mAh} / \mathrm{g})$ and the lower voltage plateau $\left(0.3 \mathrm{~V} v s \mathrm{Na}^{+} / \mathrm{Na}\right)$ make FeNCN a good candidate as an anode material for sodium ion batteries. The voltages of conversion reaction are correlated with the calculated enthalpies of formation suggesting that thermodynamics dominates the observed electrochemical conversion reaction.
\end{abstract}

\section{INTRODUCTION}

The current energy system is strongly based on fossil fuels. However, it is believed to gradually move from the use of fossil fuels to deploying renewable or nuclear energy sources. A way to compensate for the variability of energy production by renewable sources is by coupling these sources with electrical energy storage devices, such as batteries. At least in principle, batteries are able to store high energy densities and are flexible modular devices, able to use the same chemistry in a wide range of sizes by adding small cells together, enabling its design from portable to stationary applications. Up to now, lithium ion batteries have undergone a big development ${ }^{1}$, dominating especially portable applications due to the possibility of operating at room temperature. Despite sodium ion batteries delivering slightly smaller energy densities, sodium ion technology is lower cost than lithium ion batteries because of the much higher 
abundance of sodium $v s$ lithium and the possibility of using an aluminum current collector in the anode electrode (due to the lower density and price of aluminum with respect to copper).

Cathode materials in sodium ion batteries are mostly based on the same insertion chemistry as lithium ion analogues, with fluorophosphates and layered oxides being the basis of the first prototypes. However, on the anode side, the impossibility of intercalating bare sodium ions is a drawback for this technology ${ }^{2,3}$ as well as an important driver for research. In this context: high-capacity anodes such as phosphorus ${ }^{4}$ and alloys ${ }^{5}$; materials which operate at low potentials like titanates ${ }^{6}$ and hard carbon $^{7}$; and so-called environmentally friendly organic compounds, have been investigated ${ }^{8}$. In the field of metal-organic compounds, the presence of $>\mathrm{C}=\mathrm{N}$ - and $-\mathrm{C} \equiv \mathrm{N}$ - functionalities have shown to be beneficial for sodium ion batteries with polySchiff bases acting as anodes ${ }^{9}$ and Prussian Blue related systems as cathodes ${ }^{10}$. We have proposed and observed for the first time, that $-(\mathrm{N}=\mathrm{C}=\mathrm{N})-$ containing compounds are electrochemically active both for lithium and sodium ion batteries ${ }^{11}$. Transition-metal carbodiimides are metal-organic materials which have many similarities with oxides: they have the same charge (-2), similar electronegativity value, more covalency, a bit smaller electronic bandgap $^{12}$ and similar structures, but linked to transition-metal ions ${ }^{13}$ by $(\mathrm{N}=\mathrm{C}=\mathrm{N})^{2-}$ chains instead of monoatomic oxide anions. A conversion reaction has been proposed to occur when TMNCN inserts two Na ions to yield metal nanoparticles, TM, and presumably $\mathrm{Na}_{2} \mathrm{NCN}$. This fact was based on the disappearance of the reflections of the TMNCN, both by in-situ x-ray diffraction in CuNCN, as well as ex-situ XRD of other phases ${ }^{11}$, and has been confirmed by insitu XRD and Mössbauer studies of $\mathrm{FeNCN}^{14}$.

In this report, the influence of particle size on the performance of MnNCN as anode materials for sodium-ion batteries is first evaluated. Next, the voltage plateaux and the irreversibility of first cycles of this and other transition metal carbodiimides (TM= Mn, Fe, Ni or Co) are studied, evidencing that FeNCN shows the optimum performance among all the studied phases, which is correlated with its thermodynamic properties.

\section{EXPERIMENTAL}

Manganese carbodiimide was prepared by two different routes. The solid state route following the reported procedure by Liu et al. ${ }^{12}$ consists of heating a pellet of a mixture of manganese chloride and zinc carbodiimide at $600{ }^{\circ} \mathrm{C}$ under vacuum. Then, the pellet was washed with water to dissolve any residual chloride and the solution was filtered to obtain the $\mathrm{MnNCN}$ powder. Alternatively, a metathesis reaction in organic media was performed mixing lithium carbodiimide and manganese chloride in stoichiometric amounts, using THF as the solvent ${ }^{11}$ at room temperature, inside the glovebox. After stirring overnight, the solution was centrifuged and the MnNCN powder was separated from the $\mathrm{LiCl}$ which remains dissolved in THF. Lithium carbodiimide was synthesized with butyl lithium and cyanamide as precursors with a ratio of 2.4:1. However, iron, cobalt and nickel carbodiimides were obtained by synthesizing first the metal hydrogen dicyanamide in aqueous media (degassed water) under vacuum conditions (Schlenk) using the metal chlorides and cyanamide as precursors. The second step is the synthesis of the metal carbodiimide in a sealed glass ampoule. Thereby the hydrogen dicyanamides are mixed with a eutectic mixture of $\mathrm{LiCl}$ and $\mathrm{KCl}$ and heated to $400{ }^{\circ} \mathrm{C}$, yielding the iron ${ }^{15}$, cobalt and nickel ${ }^{16}$ carbodiimides. 
The powder X-ray diffractograms of the carbodiimides were collected on a Bruker D8 instrument with copper radiation $(\lambda=1.54056 \AA)$ in the $2 \theta$ range from $5-80^{\circ}$ with a step size of 0.021 for 2 hours using an air sensitive sample holder with Kapton film. For the electrochemical characterization, the measurements were carried out in CR2032 coin-cell type cells using metallic sodium as counter and reference electrodes. As the working electrode, $70 \%$ in weight of the metal carbodiimides was hand-milled for 1 hour with $20 \%$ in weight of a mixture of C65 (Timcal) and Ketjen black carbon (50:50) in order to improve the electronic conductivity and $10 \%$ in weight of polyvinylidene in $1 \mathrm{~mL}$ of $\mathrm{N}$-methyl-2-pyrrolidone fluoride as binder. As carbodiimides are not very good electronic conductors, it is very important to mix the carbon well with the materials. Then this mixture was casted by Dr. Blade technique in copper foil. Before using these electrodes, they were dried at $80{ }^{\circ} \mathrm{C}$ under vacuum overnight. Between the working and counter electrode, a Whatman glass fiber GFB/55 was used as separator. $1 \mathrm{M}$ NaFSI [Sodium bis(fluorosulfonyl)imide] (Solvionic) in methyl tetrahydrofuran (Aldrich) was selected after testing different electrolyte mixtures. Galvanostatic discharge and charge of the materials were measured in a MACCOR battery tester at $\mathrm{C} / 10$ rate between $0.005-3.0 \mathrm{~V}$ vs $\mathrm{Na}^{+} / \mathrm{Na}(\mathrm{C} / 10$ is the current needed to insert $2 \mathrm{Na}$ ions per formula unit in 10 hours).

\section{RESULTS and DISCUSSION}

The differently synthesized MnNCN were characterized by x-ray diffraction. Different diffractograms were obtained for both synthesized samples. On the one hand, following the solid state synthesis reported by X. Liu et al. ${ }^{12}$ a crystalline single phase was obtained as deduced from the narrowness of the reflections of the diffractogram and through the Rietveld refinement analysis (Figure 1a). All reflections were in good agreement with the reported trigonal space group $R \overline{3} \mathrm{mH}$ (166) reported ${ }^{17}$. On the other hand, for manganese carbodiimide synthesized in organic media, the diffractogram showed very broadened peaks due to the low crystallinity of the sample (Figure 1b). Additionally, there is a noticeable loss of intensity of the 012 reflection, which contains the $\mathrm{Mn}$ ions of adjacent $\mathrm{Mn}(\mathrm{NCN})$ layers. This could be indicative of $\mathrm{Mn}$ vacancies but is also a result of the different sample preparation for XRD data collection, which involved the use of kapton foil on top of the nanosized powders. The different crystallinity of the samples is deduced from the different narrowness of the diffraction peaks in the XRD patterns and confirmed by SEM. The particle size of the crystalline material is $4-5 \mu \mathrm{m}$ and the particles have a regular bean-like shape as can be seen in the SEM images (Figure 2a) while for the sample synthesized in organic media, the particle size was smaller (much less than $1 \mu \mathrm{m}$ ) with irregular shape (Figure 2b). 

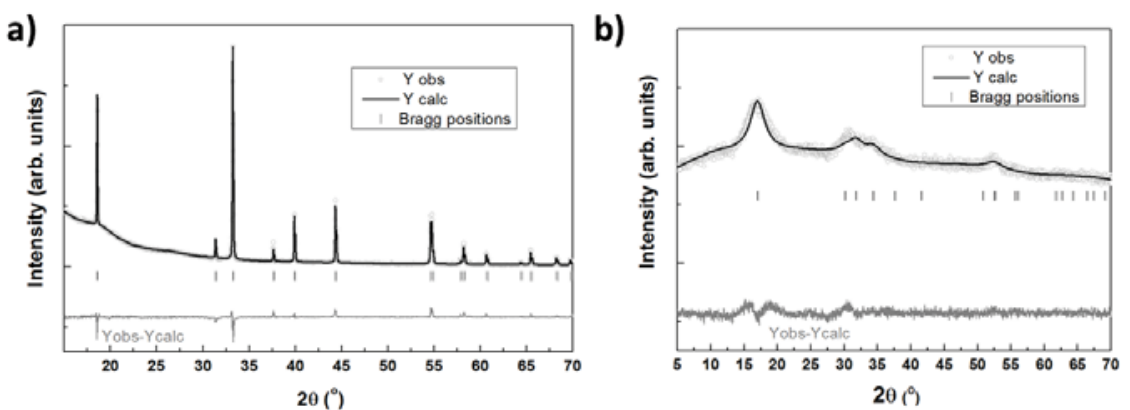

Figure 1. Rietveld refinement of MnNCN of crystalline sample (a) and the pattern matching of the sample synthesized in organic media (b). Light grey points are the experimental pattern, the black curve is the calculated pattern, dark grey line at the bottom is the difference between experimental and calculated pattern and grey lines are the corresponding reflections for the space group $R \overline{3} \mathrm{mH}$.
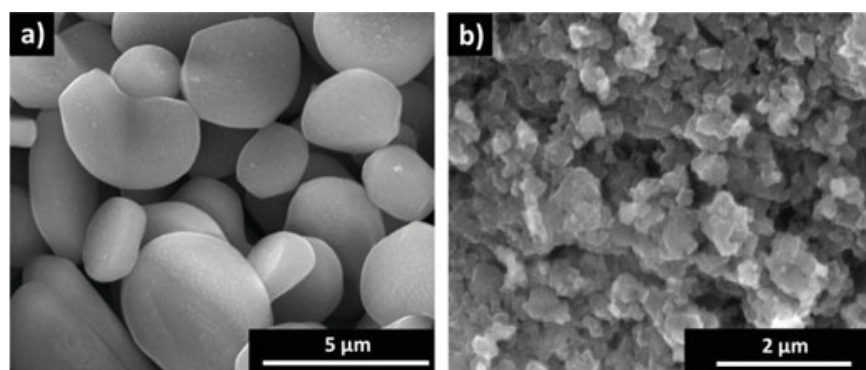

Figure 2. SEM of the different MnNCN synthesized samples where in (a) the MnNCN by the reported method and in (b) is the SEM image of the synthesized in organic media.

The X-ray powder diffractograms of iron, cobalt and nickel carbodiimides were indexed using space group $P 6_{3} / m m c$ (194), which has a NiAs-type structure. Rietveld refinement confirmed that these materials were synthesized as pure phases (as can be seen in Figure $3 \mathrm{a}$ for FeNCN as representative for the isostructural compounds) and the refined structure is drawn in Figure $3 \mathrm{~b}$. The agreement factors, cell parameters and structural characteristics obtained from the Rietveld refinements of FeNCN, CoNCN and NiNCN are presented in Table 1. 

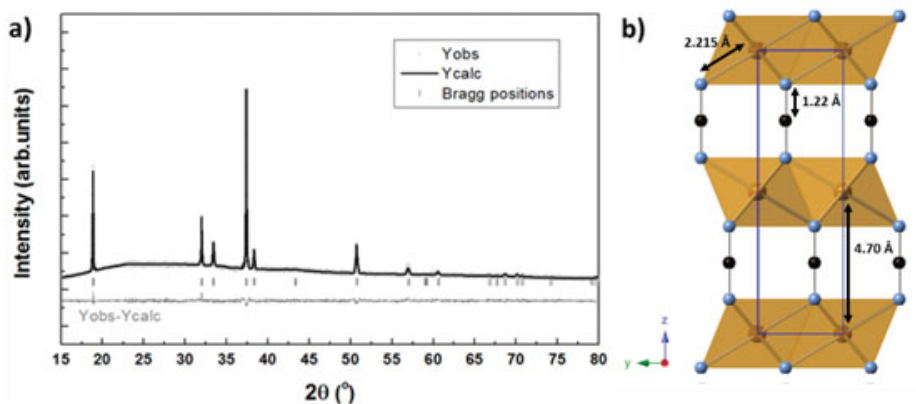

Figure 3. Observed (light grey dots), calculated (solid black line), and difference (dark grey bottom curve) patterns for the Rietveld analysis from the powder X-ray diffraction data of FeNCN and grey short vertical lines below the profile indicate the peak positions of all the possible Bragg reflections (a) and the structure of FeNCN obtained from the refinement (b).

Table 1. Space group, agreement factors, molar mass, cell parameters and structural data obtained from the Rietveld refinement method of TMNCN $\left(\mathrm{TM}=\mathrm{Mn}^{2+}, \mathrm{Fe}^{2+}, \mathrm{Co}^{2+}\right.$ or $\left.\mathrm{Ni}^{2+}\right)$, ionic radius from ref. ${ }^{18}$ and electronic configuration of the $\mathrm{TM}^{2+}$.

\begin{tabular}{|c|c|c|c|c|}
\hline & $\mathrm{MnNCN}$ & FeNCN & CoNCN & NiNCN \\
\hline Space group & $R \overline{3} m \mathrm{H}$ & $\mathrm{P}_{3} / \mathrm{mmc}$ & $\mathrm{P}_{3} / \mathrm{mmc}$ & $\mathrm{P}_{3} / \mathrm{mmc}$ \\
\hline$R_{\mathrm{p}}$ & 12.12 & 6.37 & 7.42 & 24.45 \\
\hline$R_{\mathrm{wp}}$ & 9.98 & 7.25 & 8.02 & 30.89 \\
\hline$R_{\mathrm{B}}$ & 4.36 & 2.12 & 2.98 & 7.67 \\
\hline$\chi^{2}$ & 2.92 & 1.71 & 3.59 & 4.23 \\
\hline Molar mass (g/mol) & 94.94 & 95.85 & 98.93 & 98.71 \\
\hline$a=b(\AA)$ & $3.358(3)$ & $3.280(2)$ & $3.209(3)$ & $3.158(2)$ \\
\hline$c(\AA)$ & $14.337(4)$ & $9.404(1)$ & $9.381(2)$ & $9.249(4)$ \\
\hline$r\left({ }^{\circ}\right)$ & 120 & 120 & 120 & 120 \\
\hline$V\left(\AA^{3}\right)$ & $140.037(4)$ & $87.60(1)$ & $83.64(2)$ & $79.91(6)$ \\
\hline$V / Z\left(\AA^{3}\right)$ & 46.679 & 43.8 & 41.82 & 39.96 \\
\hline TM-N (̊̊) & 2.262 & 2.215 & 2.168 & 2.119 \\
\hline Ionic radius $\mathbf{T M}^{2+}(\AA)$ & 0.83 & 0.78 & 0.745 & 0.69 \\
\hline Electronic configuration & $3 d^{5}$ & $3 d^{6}$ & $3 d^{7}$ & $3 d^{8}$ \\
\hline
\end{tabular}

\section{Electrochemical performance in sodium ion batteries}

The reduction of these transition metals to the TM (I) oxidation state seems to be quite unlikely and all $\mathrm{TM}^{2+}$ cations are expected to be reduced to $\mathrm{TM}^{0}$. At the same time, two sodium ions could link with the carbodiimide group for a full displacement reaction where two electrons would be involved. Thus, a C-rate of $1 \mathrm{C}$ was calculated as the current needed to insert 2 alkali ions per transition metal in one hour. The electrochemical performance of MnNCN, produced by different routes, in sodium ion batteries will be compared and next we extend the comparison of the performance of the three isostructural members FeNCN, CoNCN and NiNCN.

\section{i) The role of particle size in Manganese carbodiimide}

Galvanostatic discharge and charge curves of MnNCN are shown in Figure 4. Crystalline manganese carbodiimide had a very large $1^{\text {st }}$ discharge capacity $(380 \mathrm{mAh} / \mathrm{g})$, at relatively low 
potential $\left(0.35 \mathrm{~V} v s \mathrm{Na}^{+} / \mathrm{Na}\right.$ average voltage). Despite this high capacity, just $80 \mathrm{mAh} / \mathrm{g}$ were reversible in the first charge as can be seen in Figure 4a. It seemed that manganese carbodiimide material was almost electrochemically inactive. The electrochemical inactivity of MnNCN (which was previously reported by Milke et al. ${ }^{19}$ ) could be due to the proximity of the plateau to sodium electroplating. In order to see if by reducing the polarization of the electrode a low voltage plateau appeared, the common strategy of decreasing particle size was followed. This way, MnNCN synthesized in organic media was tested. A much larger reversible capacity was observed in the galvanostatic cycling of $\mathrm{MnNCN}$ synthesized in organic media, for sodium ion batteries, with three different processes being observed in the first discharge at $\sim 0.7,0.3$ and 0.1 $\mathrm{V} v s \mathrm{Na}^{+} / \mathrm{Na}$ (see Figure $4 \mathrm{~b}$ ). The smaller crystal and particle size could shorten the diffusion path of sodium ions ${ }^{20}$. As a result, the polarization could be decreased making possible the observation of electrochemical activity in $\mathrm{MnNCN}$ at voltages above Na plating.

Reaching $0.1 \mathrm{~V} v s \mathrm{Na}^{+} / \mathrm{Na}$, a plateau appeared which is likely due to the conversion reaction of manganese carbodiimide with sodium. This plateau, as expected, was very close to $0 \mathrm{~V} v \mathrm{~s}$ $\mathrm{Na}^{+} / \mathrm{Na}$, which can be the reason why it did not appear in the crystalline sample as Milke et al reported $^{19}$. The redox processes that occur during charge are clearly shown in the zoomed $\delta \mathrm{Q} \delta \mathrm{V}$ plots of the first and second cycle (Figure $4 \mathrm{c}$ and Figure 4d). On the first discharge all the redox processes are seen below $1 \mathrm{~V}$ vs $\mathrm{Na}^{+} / \mathrm{Na}$ and most of the capacity is achieved below $0.3 \mathrm{~V}$ as shown in the galvanostatic curve (not shown in the dQ/dV). In contrast, during the first and second galvanostatic charges, the two main processes where the conversion reaction was taking place are centered at $0.78 \mathrm{~V} \mathrm{vs} \mathrm{Na} / \mathrm{Na}$ (which could also have a contribution from deintercalation of $\mathrm{Na}$ ions from graphite layers) and at $2.5 \mathrm{Vvs} \mathrm{Na} / \mathrm{Na}$ the second and most relevant process, which implies a very high overpotential.
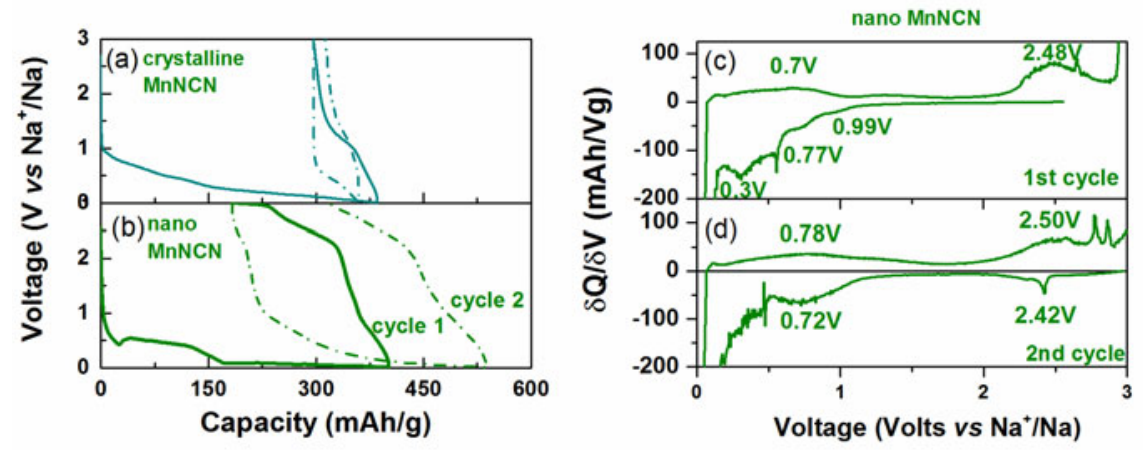

Figure 4. Galvanostatic first two cycles of crystalline $\mathrm{MnNCN}$ for sodium ion batteries in the voltage range of $0.005-3.0 \mathrm{~V} v s \mathrm{Na}^{+} / \mathrm{Na}(\mathrm{a})$, synthesized in organic media (b) and first (c) and second (d) cycle differential capacity plot of the MnNCN synthesized in organic media.

The cyclability of the crystalline sample was quite bad losing the few observed electrochemical activity in the $5^{\text {th }}$ cycle as can be seen in Figure 5a. For the less crystalline material a reversible capacity of $175 \mathrm{mAh} / \mathrm{g}$ was achieved for more than 100 cycles with a 
coulombic efficiency higher than $99 \%$ from the tenth cycle onwards (Figure 5 b). Thus the main drawback of this material to be a suitable anode for sodium ion batteries is the intrinsic overpotential that this material exhibited.
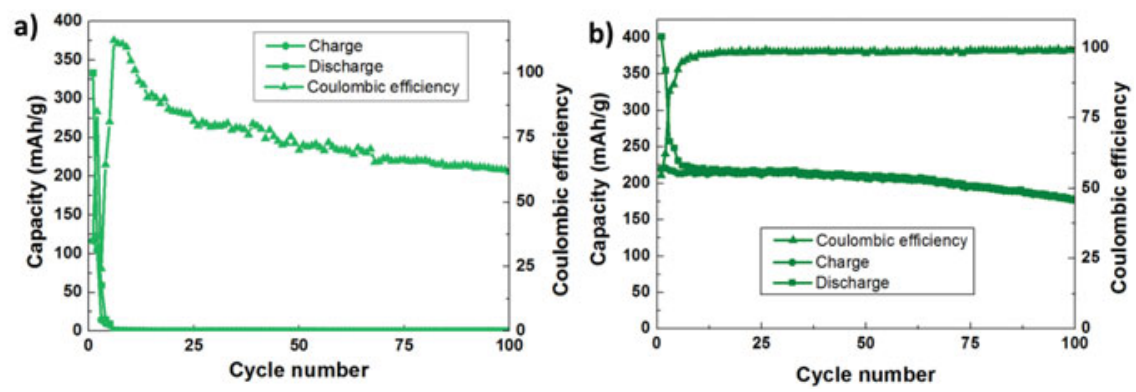

Figure 5. Cyclability of crystalline $\mathrm{MnNCN}$ (a) and $\mathrm{MnNCN}$ synthesized in organic media (b) for sodium ion batteries in the voltage range of $0.005-3.0 \mathrm{~V} v s \mathrm{Na}^{+} / \mathrm{Na}$.

Moreover, given the very low voltage of $\mathrm{Na}$ insertion $\left(0.1 \mathrm{~V} v s \mathrm{Na}^{+} / \mathrm{Na}\right)$, the material is also prone to suffer from Na plating at the electrode surface if high current densities were used and thus higher voltage materials would be preferred.

From the preliminary electrochemical measurements of $\mathrm{Mn}, \mathrm{Fe}, \mathrm{Co}$ and $\mathrm{Ni}$ carbodiimides, higher voltage of reduction was expected on increasing the number of $\mathrm{d}$ electrons on the $\mathrm{TM}^{2+}$ transition, i.e. from $\mathrm{Mn}$ to $\mathrm{Ni}^{11}$.

\section{ii) Isostructural iron, cobalt and nickel carbodiimides as anode materials in sodium ion batteries}

The electrochemical performance of FeNCN exhibited a process at $1.5 \mathrm{~V} v s \mathrm{Na}^{+} / \mathrm{Na}$ due to sodium ion adsorption into the carbon layers, providing $30 \mathrm{mAh} / \mathrm{g}$ of capacity as can be seen by the brown line in Figure 6a. At $0.8 \mathrm{~V} v s \mathrm{Na}^{+} / \mathrm{Na}$ an additional process seemed to take place, giving $150 \mathrm{mAh} / \mathrm{g}$ of irreversible capacity. This process was not observed in the published study of the electrochemistry of FeNCN performed by Sougrati et al. ${ }^{13}$. Since they used PC (Propylene Carbonate) as solvent of the electrolyte, which does not come with a high donor number, thus blocking the co-intercalation ${ }^{21}$ into the graphite interlayers, we can deduce that this process is due to co-intercalation of sodium with solvent molecules into the graphite layers. Finally, at 0.3 $\mathrm{V} v s \mathrm{Na}^{+} / \mathrm{Na}$ a large plateau of $400 \mathrm{mAh} / \mathrm{g}$ of capacity appeared which could be attributed to the conversion reaction (reduction of $\mathrm{Fe}^{2+}$ compound to metallic iron).

The galvanostatic curve of CoNCN was similar to that for FeNCN. During the first discharge, (see blue line of Figure 6a) a solid solution process appeared at $1.2 \mathrm{~V} \mathrm{vs} \mathrm{Na}^{+} / \mathrm{Na}$ which could be attributed to sodium adsorption into the carbon layer. At $0.8 \mathrm{~V} v s \mathrm{Na}^{+} / \mathrm{Na}^{+}$the sodium co-intercalation ${ }^{22}$ occurred in the same voltage as for FeNCN. At the end of the discharge, there was a plateau corresponding to the conversion reaction centered at $0.5 \mathrm{~V} v \mathrm{~s}$ $\mathrm{Na}^{+} / \mathrm{Na}$. This plateau was assigned to the reduction of CoNCN forming metallic cobalt and the 
sodium carbodiimide matrix. The $700 \mathrm{mAh} / \mathrm{g}$ of capacity delivered are a bit higher than theoretical $(542 \mathrm{mAh} / \mathrm{g})$ which could be attributed to the contribution of the carbon to the reversible capacity or to cobalt oxidation to higher than $2+$ oxidation state. In the same manner, NiNCN had almost identical galvanostatic cycling compared to $\mathrm{CoNCN}$ in terms of shape and irreversible capacity of the first cycle. During the first discharge (Figure 6a, orange line), the same processes are believed to occur. The solid solution process observed at $1.1 \mathrm{~V} v s \mathrm{Na}^{+} / \mathrm{Na}$ is likely due to the sodium adsorption into the carbon layers and the sodium co-intercalation ${ }^{21}$ with electrolyte occurs at $0.85 \mathrm{~V} v s \mathrm{Na}^{+} / \mathrm{Na}$. The plateau centered at $0.65 \mathrm{~V} v s \mathrm{Na}^{+} / \mathrm{Na}$ could be assigned to the conversion reaction where the reduction of $\mathrm{NiNCN}$ into metallic nickel and a sodium carbodiimide matrix occurred achieving a capacity of $600 \mathrm{mAh} / \mathrm{g}$. In all three cases the sharp feature observed in the $\mathrm{dQ} / \mathrm{dV}$ during discharge (Figure $6 \mathrm{~b}$ ) becomes a much broader feature upon charge which signals a different mechanism of reaction and likely different products from the starting materials at the beginning of battery assembly.
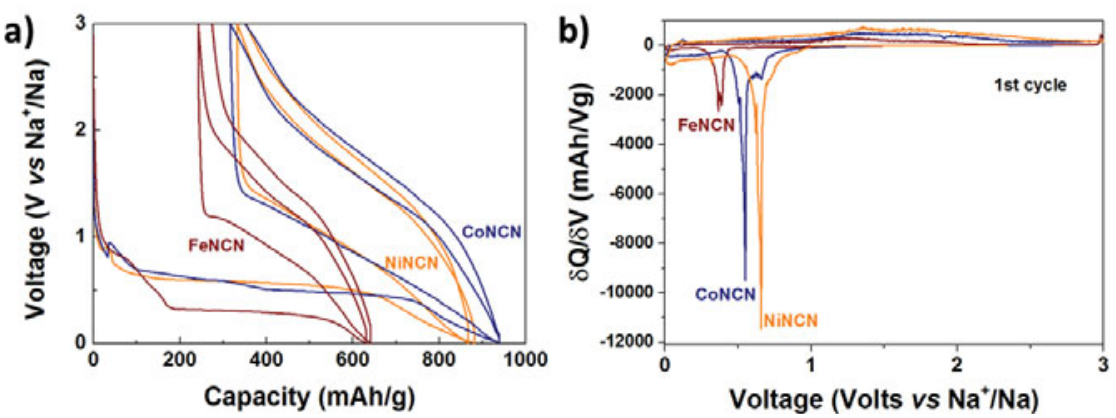

Figure 6. Galvanostatic first two cycles of FeNCN (brown), CoNCN (blue) and NiNCN (orange) (a) and the differential capacity plot of the first cycle (b).

Comparing the electrochemical performance of the described compounds, iron carbodiimide is the most suitable anode material in sodium ion batteries because of its high capacity and the smallest first cycle irreversible capacity and lowest voltage plateau among all TMNCN.

Besides the interesting electrochemical performance of FeNCN, the study of the electrochemical reduction of this series of materials is interesting from the thermodynamic point of view. Indeed, since the proposed reaction taking place in all these phases is the conversion reaction (TMNCN $+2 \mathrm{Na}^{+}+2 \mathrm{e}^{-} \rightleftarrows \mathrm{Na}_{2} \mathrm{NCN}+\mathrm{TM}$ ), it is possible to rationalize the voltage of reduction of each phase in terms of the thermodynamic properties of the reaction of formation of TMNCN from the elemental metal and $\mathrm{Na}_{2} \mathrm{NCN}$, although with opposite sign.

As is well known, the voltage of the redox reactions of the materials are related to the Gibbs free energy values according to equation 1 in which $R$ is the gas constant, $T$ the temperature, $K$ the equilibrium constant, $z$ the electron number, $F$ the Faraday constant and $E$ the electrochemical force. Thus, from the experimental voltage of reduction, $(E)$ an experimental Gibbs free energy for the conversion reaction has been calculated and is listed in Table 2. Since the products of oxidation are not confirmed to be TMNCN, the voltage of oxidation is not considered.

$$
\Delta G_{\text {reaction }}=-R T \ln K=-z F E \quad \text { (Eq. 1) }
$$


Table 2. Experimentally observed voltage of reduction of the Transition-Metal Carbodiimides in sodium ion batteries, the calculated Gibbs free energy $(\Delta G)$ from the experimental reaction voltages and the reported values for the enthalpy of formation $\left(\Delta H_{\mathrm{f}}\right)$ calculated by Launay and Dronskowski ${ }^{23}$.

\begin{tabular}{|c|c|c|c|}
\hline Material & $\begin{array}{c}\text { Voltage } \\
V v s \mathrm{Na}^{+} / \mathrm{Na}\end{array}$ & $\underset{k J / \mathrm{mol}}{\Delta \mathbf{G}}$ & $\begin{array}{c}\Delta \mathbf{H}_{\mathbf{f}} \\
\mathrm{kJ} / \mathrm{mol}\end{array}$ \\
\hline $\mathrm{MnNCN}$ & 0.2 & -38.6 & +44.8 \\
\hline FeNCN & 0.4 & -77.2 & +77.3 \\
\hline $\mathrm{CoNCN}$ & 0.5 & -96.5 & +101.5 \\
\hline NiNCN & 0.65 & -125.45 & +123.8 \\
\hline
\end{tabular}

Given the recent synthesis of transition-metal carbodiimides (first reported in 2005$)^{13}$, there are no experimental reports on measured Gibbs free energies of formation, but a first-principles theoretical study had predicted the enthalpy of formation for TMNCN $\left(\mathrm{TM}=\mathrm{Mn}^{2+}, \mathrm{Fe}^{2+}, \mathrm{Co}^{2+}\right.$ or $\mathrm{Ni}^{2+}$ ) from their elements ${ }^{23}$ ahead of synthesis. These values, also listed in Table 2, predicted that these metastable compounds could not be synthesized from their starting elements, so other routes (described above) were followed. If we now consider that there is a constant term for the formation of $\mathrm{Na}_{2} \mathrm{NCN}$ that we are neglecting for every material, and since the contribution of the entropy term to the Gibbs free energy is usually much lower compared to the enthalpy term at room temperature $(T \Delta S \ll \Delta H)$, the enthalpy values can be directly compared to the Gibbs energy of each transition-metal carbodiimide as given in Eq. 2:

$$
\Delta G_{\text {reaction }}=\Delta H-T \Delta S \approx \Delta H \quad(\text { Eq. 2) }
$$

Indeed, by comparing the two columns on the right in Table 2, a very good correlation is observed between the here reported Gibbs free energies of the conversion reaction and the calculated enthalpies of formation of the TMNCN. Therefore, despite that these calculations have one experimental error which is based on the intrinsic overpotential that these materials show in their electrochemical behavior and which in materials that operate through conversion reactions can be very large (the experimentally observed voltage being lower than the thermodynamic expected one) and despite a theoretical uncertainty in the theoretical formation enthalpies due to the used exchange-correlation functional (GGA, Perdew-Wang 91) plus other simplifications, the first reduction reaction in these systems seems to be dominated by the thermodynamics of the reaction. 


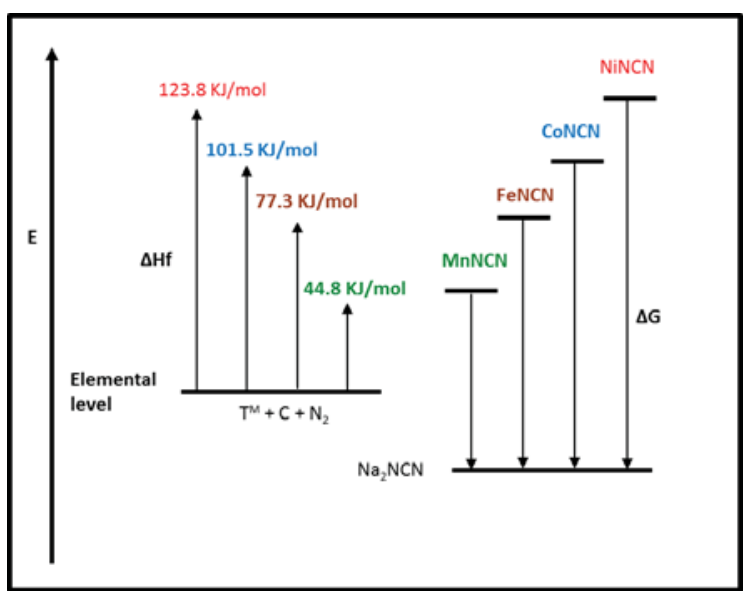

Figure 7. Scheme of the thermodynamic studies of MnNCN, FeNCN, CoNCN and NiNCN and the relationship with $\mathrm{Na}_{2} \mathrm{NCN}$ compound.

A scheme summarizing these correlations (Figure 7) suggests a negative enthalpy of formation of $\mathrm{Na}_{2} \mathrm{NCN}$ from its elements as well as the spontaneous reaction observed in the electrochemical cells when TMNCN are left to react with sodium. Although there seems to be no experimental value for the enthalpy of formation of $\mathrm{Na}_{2} \mathrm{NCN}$, the related alkaline-earth phase $\mathrm{CaNCN}$ is indeed exothermic with an enthalpy of formation of $-350.6 \mathrm{~kJ} / \mathrm{mol}^{24}$.

\section{CONCLUSIONS}

The electrochemical performance of conversion-based anode materials TMNCN $(\mathrm{TM}=$ $\mathrm{Mn}, \mathrm{Fe}, \mathrm{Co}, \mathrm{Ni}$ ) have been investigated for sodium ion batteries. MnNCN, which has a very low voltage for $\mathrm{Na}$ insertion of $\sim 0.1 \mathrm{~V} v s \mathrm{Na}^{+} / \mathrm{Na}$ shows a strong dependence of its electrochemical performance on particle size according to SEM, with micron-sized MnNCN being almost inactive and nano-sized $\mathrm{MnNCN}$ synthesized in organic media showing at least half of the theoretical capacity for the conversion reaction. FeNCN, CoNCN and NiNCN show much higher reversible capacity and lower overpotential. TMNCN improve the energy density and efficiency in comparison with oxides due to the lower voltage where the conversion reaction is taking place. CoNCN and NiNCN exhibited large capacities in the first cycle consisting of a 2econversion process. Among all these TMNCN, FeNCN shows the smallest first cycle irreversible capacity $(220 \mathrm{mAh} / \mathrm{g})$ and a lower suitable voltage plateau $\left(0.3 \mathrm{~V} v s \mathrm{Na}^{+} / \mathrm{Na}\right)$. This makes FeNCN a good candidate as an anode material for sodium ion batteries. The voltages of the conversion reaction in the first cycle, as deduced from the $\delta \mathrm{Q} / \delta \mathrm{V}$, increases with the number of $d$ electrons. The corresponding Gibbs free energies of the conversion reaction deduced from the voltage are in good agreements with the calculated enthalpies of formation of the TMNCN, suggesting that thermodynamics dominates the conversion reaction. 


\section{ACKNOWLEDGMENTS}

The authors thank Prof. Adelhelm for fruitful discussions on the thermodynamic calculations. AEB thanks UPV for a FPI-UPV PhD Fellowship. Financial support of FEDER funds through the Spanish Ministry MINECO MAT-2013-41128-R project granted to the University of Basque Country and ENE2013-44330-R granted to CIC Energigune is acknowledged.

\section{REFERENCES}

1. J.-M. Tarascon and M. Armand "Issues and challenges facing rechargable lithium batteries" Nature, 2001, 414, 359-367.

2. V. Palomares, P. Serras, I. Villaluenga, K. B. Hueso, J. Carretero-Gonzalez and T. Rojo "Naion batteries, recent advances and present challenges to become low cost energy storage systems." Energy Environ. Sci. 2012, 5, 5884-5901.

3. V. Palomares, M. Casas-Cabanas, E. Castillo-Martínez, M. H. Han and T. Rojo, "Update on Na-based battery materials. A growing research path", Energy Environ. Sci., 2013, 6, 23122337.

4. Y. Kim, Y. Park, A. Choi, N. S. Choi, J. Kim, J. Lee, J. H. Ryu, S. M. Oh and K. T. Lee, “An Amorphous Red Phosphorus/ Carbon Composite as a Promising Anode Material for Sodium Ion Batteries." Adv. Mater. 2013, 25, 3045-3049.

5. S. Komaba, Y. Matsuura, T. Ishikawa, N. Yabuuchi, W. Murata and S. Kuze, "Redox reaction of Sn-polyacrylate electrodes in aprotic Na cell." Electrochem.Commun. 2012, 21, 65-68.

6. P. Senguttuvan, G. Rousse, V. Seznec, J.-M. Tarascon and R. Palacin, "Na2Ti3O7: Lowest Voltage Ever Reported Oxide Insertion Electrode for Sodium Ion Batteries" Chemistry of Materials, 2011, 23, 4109-4111.

7. Zhao J., Zhao L., Chihara K., Okada S., Yamaki J.i., Matsumoto S., Kuze S. and Nakane K., "Electrochemical and thermal properties of hard carbon-type anodes for Na-ion batteries" $J$.

Power Sources, 2013, 244, 752-757.

8. L. Zhao, J. M. Zhao, Y. -S. Hu, H. Li, Z. B. Zhou, M. Armand and L. Q. Chen, "Disodium Terephthalate (Na2C8H4O4) as High Performance Anode Material for Low-Cost RoomTemperature Sodium-Ion Battery" Adv. Energy Mater., 2012, 2, 962-965.

9. E. Castillo-Martinez, J. Carretero-Gonzalez and M. Armand; "Polymeric Schiff Bases as LowVoltage Redox Centers for Sodium-Ion Batteries" Angew. Chem. Int. Ed. 2014, 54, 5445-5449. 10. Y. Lu, L. Wang, J. Cheng and J.B. Goodenough; "Prussian blue: a new framework of electrodematerials for sodium batteries" Chem. Commun., 2012, 48, 6544-6546.

11. A. Eguía-Barrio, E. Castillo-Martínez, X. Liu, R. Dronskowski, M. Armand and T. Rojo; "Carbodiimides: new materials applied as anode electrodes for sodium and lithium ion batteries" J. Mater. Chem. A 2016, 4, 1608-1611.

12. T. D. Boyko, R. J. Green, R. Dronskowski and A. Moewes; "Electronic Band Gap Reduction in Manganese Carbodiimide: MnNCN"; J. Phys. Chem. C 2013, 117, 12754. 
13. X. Liu, M. Krott, P. Müller, C. Hu, H. Lueken and R. Dronskowski; "Synthesis, Crystal Structure, and Properties of MnNCN, the First Carbodiimide of a Magnetic Transition Metal"; Inorg. Chem.; 2005, 44 (9), 3001.

14. M. T. Sougrati, A. Darwiche, X. Liu, A. Mahmoud, R. P. Hermann, S. Jouen, L. Monconduit, R. Dronskowski and L. Stievano; "Transition-Metal Carbodiimides as Molecular Negative Electrode Materials for Lithium- and Sodium-Ion Batteries with Excellent Cycling Properties" Angew. Chem. Int. Ed. 2016, 55, 5090-5095.

15. X. Liu, L. Stork, M. Speldrich, H. Lueken and R. Dronskowski; "FeNCN and Fe(NCNH $)_{2}$ : Synthesis, Structure, and Magnetic Properties of a Nitrogen-Based Pseudo-oxide and -hydroxide of Divalent Iron"; Chem. Eur. J. 2009, 15, 1558.

16. M. Krott, X. Liu, B. P. T. Fokwa, M. Speldrich, H. Lueken and R. Dronskowski; "Synthesis, Crystal-Structure Determination and Magnetic Properties of Two New Transition-Metal Carbodiimides: CoNCN and NiNCN"; Inorg. Chem. 2007, 46, 2204.

17. M. Krott, A. Houben, P. Müller, W. Schweika and R. Dronskowski; "Determination of the magnetic structure of manganese carbodiimide with diffraction experiments using polarized neutrons "; Phys. Rev. B; 2009, 80, 024117.

18. L. H. Ahrens; "The use of ionization potentials Part 1. Ionic radii of the elements"; Geochim. Cosmochim. Acta, 1952, 2 (3), 155.

19. B. Milke, C. Wall, S. Metzke, G. Clavel, M. Fichtner and C. Giordano; "A simple synthesis of MnN0.43@C nanocomposite: characterization and application as battery material"; J. Nanopart. Res. 2014, 16, 2795.

20. J. Cui, C. Qing, Q. Zhang, C. Su, X. Wang, B. Yang and X. Huang; "Effect of the particle size on the electrochemical performance of nano-Li2FeSiO4/C composites" Ionics 2014, 20, 23.

21 B. Jache and P. Adelhelm, "Use of Graphite as a Highly Reversible Electrode with Superior Cycle Life for Sodium-Ion Batteries by Making Use of Co-Intercalation Phenomena"; Angew. Chem. Int. Ed., 2014, 53 (38), 10169.

22. B. Jache, J. O. Binder, T. Abe and P. Adelhelm; "A comparative study on the impact of different glymes and their derivatives as electrolyte solvents for graphite co-intercalation electrodes in lithium-ion and sodium-ion batteries"; Phys. Chem. Chem. Phys., 2016, 18, 14299.

23. M. Launay and R. Dronskowski, "A Theoretical Study on the Existence and Structures of Some Hypothetical First-Row Transition-Metal M(NCN) compounds" Z. Naturforsch. 2005, 60b, 437.

24 R. Blachnik: Elemente, anorganische Verbindungen und Materialen, Minerale; J. D’Ans, E. Lax: Taschenbuch für Chemiker und Physiker; Springer-Verlag, Berlin, Heidelberg, New York (1998). 